\title{
Field Representations in General Gyrotropic Media in Spherical Coordinates
}

\author{
Cheng-Wei Qiu, Student Member, IEEE, Le-Wei Li, Fellow, IEEE, Qun Wu, Senior Member, IEEE, and \\ Tat-Soon Yeo, Fellow, IEEE
}

\begin{abstract}
This letter presents a field expansion of the solution to three-dimensional electromagnetic problems in a material characterized by the general gyrotropic media. Both $\bar{\epsilon}$ and $\bar{\mu}$ tensors are gyrotropic, specified by six components and considered in the present analysis under spherial coordinates. Electromagnetic fields in general gyrotropic media are solved by using the method of separation of variables. Much effort is paid to the solution of a coupled set of second-order differential equations. Some specific situations of the parameters are investigated in detail. By properly grouping some terms, the electromagnetic fields are represented in a compact form. Compared to the existing results in the spherical coordinate system, the material properties investigated in this letter are more general. The general expressions of the fields are found to be reducible to those existing results for simplier cases.
\end{abstract}

Index Terms-Field representations, gyrotropic media, spherical coordinates.

\section{INTRODUCTION}

$\mathbf{R}$ EPRESENTATION of electromagnetic (EM) fields in gyrotropic media (where both permittivity tensor $\overline{\boldsymbol{\epsilon}}$ and permeability tensor $\overline{\boldsymbol{\mu}}$ have six nonzero elements) has been an important topic in electromagnetic theory. This is especially true when the electromagnetic fields are described in spherical coordinates. In spherical coordinate systems, such a material is characterized by six tensor elements (three for permittivity and three for permeability) and is inhomogeneous since the rotational symmetry imposes an origin and constraints the material principal optical axes to be orthogonal to the surface of a sphere or conformal with it.

Due to the advances in material science and technology which have manifested fabrications of various kinds of gyrotropic materials, considerable attention has been paid in recent years to the interactions of electromagnetic waves with gyrotropic materials [1]-[5]. Some other works developed certain methods such as scalar Hertz potentials [6] and scalar superpotentials [7]. Due to the complexity in expression forms of the electromagnetic fields in spherical coordinate systems, the formulations of EM fields are tedious, lengthy, and complicated. The relevant works [8], [9] studied only electromagnetic waves and interactions in gyrotropic media in spherical coordinates and the materials have only electric gyrotropy, though. Other works dealt with the fields

Manuscript received June 15, 2004; revised November 15, 2005.

C.-W. Qiu, L.-W. Li, and T.-S. Yeo are with the Department of Electrical and Computer Engineering, National University of Singapore, Kent Ridge, Singapore 119260, Singapore (e-mail: elelilw@nus.edu.sg; eleyeots@nus.edu.sg).

Q. Wu is with the Department of Electronics and Communications Engineering, Harbin Institute of Technology, Harbin 150001, China (e-mail: qwu@hit.edu.cn).

Digital Object Identifier 10.1109/LAWP.2005.862055 and eigenvalues either in uniaxial anisotropic media [10] or in the Cartesian and cylindrical coordinate systems [11], where the formulations are much simplier than what follows in the current work. It appears that no other work is available in the literature to represent, in the present way, the EM fields in this kind of materials containing both electric and magnetic gyrotropies.

In this work, we study a kind of general gyrotropic media which are characterized by constitutive tensors of permittivity and permeability in the following forms:

$$
\begin{aligned}
& \overline{\boldsymbol{\epsilon}}=\left[\begin{array}{ccc}
\epsilon_{r} & 0 & 0 \\
0 & \epsilon_{\tau} & \epsilon_{\sigma} \\
0 & -\epsilon_{\sigma} & \epsilon_{\tau}
\end{array}\right] \\
& \overline{\boldsymbol{\mu}}=\left[\begin{array}{ccc}
\mu_{r} & 0 & 0 \\
0 & \mu_{\gamma} & \mu_{\zeta} \\
0 & -\mu_{\zeta} & \mu_{\gamma}
\end{array}\right]
\end{aligned}
$$

where the identity dyadic is expressed as $\bar{I}=\hat{r} \hat{r}+\hat{\theta} \hat{\theta}+\hat{\phi} \hat{\phi}$.

In the present study, much effort is made toward solving the second-order differential equations and then obtaining all the electric and magnetic field components of TE and TM waves with respect to $\widehat{\mathbf{r}}$. Different cases, both specific and general, are considered. In addition, this letter makes some corrections of some of the field expressions in [8] when the solution is reduced to that of the simplier case discussed in [8].

\section{BASIC FORMULATIONS}

For anisotropic media, the constitutive relationships as used in the Maxwell equations are given below

$$
\begin{aligned}
\nabla \times \boldsymbol{E} & =i \omega \overline{\boldsymbol{\mu}} \cdot \boldsymbol{H} \\
\nabla \times \boldsymbol{H} & =-i \omega \overline{\boldsymbol{\epsilon}} \cdot \boldsymbol{E}+\boldsymbol{J}
\end{aligned}
$$

where the time dependence $e^{-i \omega t}$ has been assumed but suppressed.

To solve the Maxwell's equations, it is more convenient to pose the problem in terms of only the radial functions $E_{r}$ and $H_{r}$ [8]. After a somewhat lengthy but careful algebra manipulation, we arrive at a coupled set of differential equations involving only radial components $E_{r}$ and $H_{r}$

$$
\begin{aligned}
\frac{1}{r^{2}} & \frac{\partial^{2}\left(r^{2} E_{r}\right)}{\partial r^{2}}+\frac{\epsilon_{\tau}}{\epsilon_{r}} \frac{1}{r^{2} \sin \theta} \frac{\partial\left(\sin \theta \frac{\partial E_{r}}{\partial \theta}\right)}{\partial \theta} \\
& +\frac{\epsilon_{\tau}}{\epsilon_{r}} \frac{1}{r^{2} \sin ^{2} \theta} \frac{\partial^{2} E_{r}}{\partial \phi^{2}}+\omega^{2} \frac{\epsilon_{\tau}}{\mu_{\gamma}}\left(\mu_{\gamma}^{2}+\mu_{\zeta}^{2}\right) E_{r} \\
& -i \omega \frac{\mu_{r}}{\mu_{\gamma} \epsilon_{r}}\left(\epsilon_{\sigma} \mu_{\gamma}+\epsilon_{\tau} \mu_{\zeta}\right) \frac{1}{r^{2}} \frac{\partial\left(r^{2} H_{r}\right)}{\partial r}=0
\end{aligned}
$$




$$
\begin{aligned}
\frac{1}{r^{2}} & \frac{\partial^{2}\left(r^{2} H_{r}\right)}{\partial r^{2}}+\frac{\mu_{\gamma}}{\mu_{r}} \frac{1}{r^{2} \sin \theta} \frac{\partial\left(\sin \theta \frac{\partial H_{r}}{\partial \theta}\right)}{\partial \theta} \\
& +\frac{\mu_{\gamma}}{\mu_{r}} \frac{1}{r^{2} \sin ^{2} \theta} \frac{\partial^{2} H_{r}}{\partial \phi^{2}}+\omega^{2} \frac{\mu_{\gamma}}{\epsilon_{\tau}}\left(\epsilon_{\tau}^{2}+\epsilon_{\sigma}^{2}\right) H_{r} \\
& -i \omega \frac{\epsilon_{r}}{\epsilon_{\tau} \mu_{r}}\left(\epsilon_{\sigma} \mu_{\gamma}+\epsilon_{\tau} \mu_{\zeta}\right) \frac{1}{r^{2}} \frac{\partial\left(r^{2} E_{r}\right)}{\partial r}=0 .
\end{aligned}
$$

From the properties of the associated Legendre polynomials, we will have

$$
\frac{1}{r^{2} \sin \theta} \frac{\partial}{\partial \theta}\left(\sin \theta \frac{\partial}{\partial \theta} \bullet\right)+\frac{1}{r^{2} \sin ^{2} \theta} \frac{\partial^{2}}{\partial \phi^{2}} \bullet=-v(v+1) \bullet
$$

where the bullet $\bullet$ denotes the separated part of either $E_{r}$ or $H_{r}$ according to a specific case, but it should be, however, conformal through (4). Certainly, this will lead to the Legendre polynomial as its solution.

Using the method of separation of variables, it is found that the solutions are composed of superpositions of spherical Bessel functions, associated Legendre polynomials, and harmonic functions, which is similar to that in [8]

$$
\left[\begin{array}{l}
E_{r} \\
H_{r}
\end{array}\right]=\sum_{m, n}\left[\begin{array}{c}
b_{o}^{e} m n E_{n} \\
a_{o}^{e} m n
\end{array} H_{n}\right] P_{n}^{m}(\cos \theta){ }_{\sin }^{\cos } m \phi
$$

where the forms of $E_{n}$ and $H_{n}$ depend on the specified cases discussed later.

For the neat and simplicity of the further derivations, let us introduce some identities first

$$
z_{v}^{(q)}(\kappa r)=\left\{\begin{array}{ll}
j_{v}(\kappa r), & q=1 \\
y_{v}(\kappa r), & q=2 \\
h_{v}^{(1)}(\kappa r), & q=3 \\
h_{v}^{2}(\kappa r), & q=4
\end{array} .\right.
$$

In (6), $j_{v}(\kappa r)$ and $y_{v}(\kappa r)$ denote spherical Bessel functions of the first kind and the second kind, respectively. The $h_{v}^{(1)}(\kappa r)$ and $h_{v}^{(2)}(\kappa r)$ represent spherical Hankel functions of the first kind and the second kind, respectively. The order $v$, when used to describe the waves and fields in isotropic media, is usually an integer; but it is not necessarily an integer for characterize waves in gyrotropic media. In this case, it depends on the medium parameters and has two values

$$
\begin{aligned}
& v_{1}\left(v_{1}+1\right)=n(n+1) \frac{\epsilon_{\tau}}{\epsilon_{r}} \\
& v_{2}\left(v_{2}+1\right)=n(n+1) \frac{\mu_{\gamma}}{\mu_{r}} .
\end{aligned}
$$

\section{Field RePRESENTAIONS IN DifFerent CASES}

In the last section, the eigenvalues and the angular functions were obtained. In this section, much effort will be made on (3) dealing with the radial functions. Subsequently, we will solve (3) for different cases.
A. Case 1: $\epsilon_{\sigma} \mu_{\gamma}+\epsilon_{\tau} \mu_{\zeta}=0\left(\epsilon_{\sigma} \neq 0\right.$ and $\left.\mu_{\zeta} \neq 0\right)$

In this case, the (3) is decoupled into

$$
\begin{aligned}
& \frac{1}{r^{2}} \frac{\partial^{2}\left(r^{2} E_{r}\right)}{\partial r^{2}}+\frac{\epsilon_{\tau}}{\epsilon_{r}} \frac{1}{r^{2} \sin \theta} \frac{\partial\left(\sin \theta \frac{\partial E_{r}}{\partial \theta}\right)}{\partial \theta} \\
& +\frac{\epsilon_{\tau}}{\epsilon_{r}} \frac{1}{r^{2} \sin ^{2} \theta} \frac{\partial^{2} E_{r}}{\partial \phi^{2}}+\omega^{2} \frac{\epsilon_{\tau}}{\mu_{\gamma}}\left(\mu_{\gamma}^{2}+\mu_{\zeta}^{2}\right) E_{r}=0 \\
& \frac{1}{r^{2}} \frac{\partial^{2}\left(r^{2} H_{r}\right)}{\partial r^{2}}+\frac{\mu_{\gamma}}{\mu_{r}} \frac{1}{r^{2} \sin \theta} \frac{\partial\left(\sin \theta \frac{\partial H_{r}}{\partial \theta}\right)}{\partial \theta} \\
& \quad+\frac{\mu_{\gamma}}{\mu_{r}} \frac{1}{r^{2} \sin ^{2} \theta} \frac{\partial^{2} H_{r}}{\partial \phi^{2}}+\omega^{2} \frac{\mu_{\gamma}}{\epsilon_{\tau}}\left(\epsilon_{\tau}^{2}+\epsilon_{\sigma}^{2}\right) H_{r}=0
\end{aligned}
$$

Substitute (5) into (8) and after some lengthy manipulations, we obtain

$$
\begin{aligned}
& E_{n}=\frac{\alpha_{t_{1}}}{r} z_{v_{1}}^{(q)}\left(k_{t_{1}} r\right) \\
& H_{n}=-i \eta_{r}^{-2} \eta_{t} \frac{\alpha_{t_{2}}}{r} z_{v_{2}}^{(q)}\left(k_{t_{2}} r\right)
\end{aligned}
$$

where

$$
\begin{aligned}
& \alpha_{t_{1}}=k_{r}^{-\frac{3}{2}} \sqrt{\frac{2 k_{t_{1}}}{\pi}} \\
& \alpha_{t_{2}}=k_{r}^{-\frac{3}{2}} \sqrt{\frac{2 k_{t_{2}}}{\pi}} .
\end{aligned}
$$

In (9) and (10), we have the following interparameters:

$$
\begin{aligned}
k_{t_{1}}^{2} & =\omega^{2} \frac{\epsilon_{\tau}}{\mu_{\gamma}}\left(\mu_{\gamma}^{2}+\mu_{\zeta}^{2}\right) \\
k_{t_{2}}^{2} & =\omega^{2} \frac{\mu_{\gamma}}{\epsilon_{\tau}}\left(\epsilon_{\tau}^{2}+\epsilon_{\sigma}^{2}\right) \\
k_{r}^{2} & =\omega^{2} \mu_{r} \epsilon_{r} \\
\eta_{r} & =\sqrt{\frac{\mu_{r}}{\epsilon_{r}}} \\
\eta_{t} & =\sqrt{\frac{\mu_{\gamma}}{\epsilon_{\tau}}} .
\end{aligned}
$$

In order to obtain the complete field representation, the tangential components of electromagnetic fields are needed and they are expressed as follows:

$$
\begin{aligned}
\frac{1}{r \sin \theta} \frac{\partial}{\partial \phi} E_{r}-\frac{1}{r} \frac{\partial}{\partial r}\left(r E_{\phi}\right) & =i \omega \mu_{\gamma} H_{\theta}+i \omega \mu_{\zeta} H_{\phi} \\
\frac{1}{r} \frac{\partial}{\partial \phi}\left(r E_{\theta}\right)-\frac{1}{r} \frac{\partial}{\partial \theta} E_{r} & =-i \omega \mu_{\zeta} H_{\theta}+i \omega \mu_{\gamma} H_{\phi}
\end{aligned}
$$


and

$$
\begin{aligned}
\frac{1}{r \sin \theta} \frac{\partial}{\partial \phi} H_{r}-\frac{1}{r} \frac{\partial}{\partial r}\left(r H_{\phi}\right) & =-i \omega \epsilon_{\tau} E_{\theta}-i \omega \epsilon_{\theta} E_{\phi} \\
\frac{1}{r} \frac{\partial}{\partial \phi}\left(r E_{\theta}\right)-\frac{1}{r} \frac{\partial}{\partial \theta} E_{r} & =i \omega \epsilon_{\sigma} E_{\theta}-i \omega \epsilon_{\tau} E_{\phi}
\end{aligned}
$$

To make the solution more straightforward, we need to separate the field components into TE- and TM-field modes subsequently with respect to $\widehat{\boldsymbol{r}}$.

1) TE-field Modes to $\widehat{\boldsymbol{r}} .:$ For TE-field modes, (12) is reduced to

$$
\begin{aligned}
-\frac{1}{r} \frac{\partial}{\partial r}\left(r E_{\phi}^{T E}\right) & =i \omega \mu_{\gamma} H_{\theta}^{T E}+i \omega \mu_{\zeta} H_{\phi}^{T E} \\
& \frac{1}{r} \frac{\partial}{\partial r}\left(r E_{\theta}^{T E}\right)=-i \omega \mu_{\zeta} H_{\theta}^{T E}+i \omega \mu_{\gamma} H_{\phi}^{T E} .
\end{aligned}
$$

Substituting (14) into (13), we have

$$
\begin{aligned}
& \frac{\partial^{2}}{\partial r^{2}}\left(r E_{\theta}^{T E}\right)+k_{t_{2}}^{2}\left(r E_{\theta}^{T E}\right) \\
& \quad=i \omega \mu_{\gamma} \frac{1}{\sin \theta} \frac{\partial}{\partial \phi} H_{r}-i \omega \mu_{\zeta} \frac{\partial}{\partial \theta} H_{r} \\
& \frac{\partial^{2}}{\partial r^{2}}\left(r E_{\phi}^{T E}\right)+k_{t_{2}}^{2}\left(r E_{\phi}^{T E}\right) \\
& \quad=-i \omega \mu_{\zeta} \frac{1}{\sin \theta} \frac{\partial}{\partial \phi} H_{r}-i \omega \mu_{\gamma} \frac{\partial}{\partial \theta} H_{r} .
\end{aligned}
$$

After careful manipulations, we finally obtain

$$
\begin{aligned}
& E_{\theta}^{T E}=\mp \omega \eta_{r}^{-2} \eta_{t} \alpha_{t_{2}} \sum_{m, n} \frac{a_{o}^{e} m n}{v_{2}\left(v_{2}+1\right)} z_{v_{2}}^{(q)}\left(k_{t_{2}} r\right) \\
& \times\left[\mu_{\gamma} \frac{m}{\sin \theta} P_{n}^{m}(\cos \theta){ }_{\cos }^{\sin } m \phi\right. \\
& \left. \pm \mu_{\zeta} \frac{d P_{n}^{m}(\cos \theta)}{d \theta} \sin m \phi\right] \\
& E_{\phi}^{T E}=\mp \omega \eta_{r}^{-2} \eta_{t} \alpha_{t_{2}} \sum_{m, n} \frac{a_{o}^{e} m n}{v_{2}\left(v_{2}+1\right)} z_{v_{2}}^{(q)}\left(k_{t_{2}} r\right) \\
& \times\left[-\mu_{\zeta} \frac{m}{\sin \theta} P_{n}^{m}(\cos \theta){ }_{\cos }^{\sin } m \phi\right. \\
& \left. \pm \mu_{\gamma} \frac{d P_{n}^{m}(\cos \theta)}{d \theta} \sin _{\sin }^{\cos } m \phi\right] \\
& H_{\theta}^{T E}=-i \eta_{r}^{-2} \eta_{t} \alpha_{t_{2}} \sum_{m, n} \frac{a_{o}^{e} m n}{v_{2}\left(v_{2}+1\right)} \frac{\partial\left[r z_{v_{2}}^{(q)}\left(k_{t_{2}} r\right)\right]}{r \partial r} \\
& \times \frac{d P_{n}^{m}(\cos \theta)}{d \theta} \sin _{\sin }^{\cos } m \phi \\
& H_{\phi}^{T E}= \pm i \eta_{r}^{-2} \eta_{t} \alpha_{t_{2}} \sum_{m, n} \frac{a_{o}^{e} m n}{v_{2}\left(v_{2}+1\right)} \frac{m}{\sin \theta} \frac{\partial\left[r z_{v_{2}}^{(q)}\left(k_{t_{2}} r\right)\right]}{r \partial r} \\
& \times P_{n}^{m}(\cos \theta){ }_{\cos }^{\sin } m \phi \text {. }
\end{aligned}
$$

2) TM-field Modes to $\widehat{\boldsymbol{r}}$ : For TM-field modes, (13) is similarly rewritten as

$$
\begin{aligned}
-\frac{1}{r} \frac{\partial}{\partial r}\left(r H_{\phi}^{T M}\right) & =-i \omega \epsilon_{\tau} E_{\theta}^{T M}-i \omega \epsilon_{\sigma} E_{\phi}^{T M} \\
\frac{1}{r} \frac{\partial}{\partial r}\left(r H_{\theta}^{T M}\right) & =i \omega \epsilon_{\sigma} E_{\theta}^{T M}-i \omega \epsilon_{\tau} E_{\phi}^{T M} .
\end{aligned}
$$

Substituting (17) into (12), we have

$$
\begin{aligned}
& \frac{\partial^{2}\left(r H_{\theta}^{T M}\right)}{\partial r^{2}}+k_{t_{1}}^{2}\left(r H_{\theta}^{T M}\right) \\
& =-i \omega \epsilon_{\tau} \frac{1}{\sin \theta} \frac{\partial E_{r}}{\partial \phi}+i \omega \epsilon_{\sigma} \frac{\partial E_{r}}{\partial \theta} \\
& \frac{\partial^{2}\left(r H_{\phi}^{T M}\right)}{\partial r^{2}}+k_{t_{1}}^{2}\left(r H_{\phi}^{T M}\right) \\
& =-i \omega \epsilon_{\sigma} \frac{1}{\sin \theta} \frac{\partial E_{r}}{\partial \phi}+i \omega \epsilon_{\tau} \frac{\partial E_{r}}{\partial \theta} .
\end{aligned}
$$

Again after careful manipulations, we finally have

$$
\begin{aligned}
& H_{\theta}^{T M}= \pm i \omega \alpha_{t_{1}} \sum_{m, n} \frac{b_{o}^{b_{o}} m n}{v_{1}\left(v_{1}+1\right)} z_{v_{1}}^{(q)}\left(k_{t_{1}} r\right) \\
& \times\left[\epsilon_{\tau} \frac{m}{\sin \theta} P_{n}^{m}(\cos \theta){ }_{\cos }^{\sin } m \phi\right. \\
& \left.\mp \epsilon_{\sigma} \frac{d P_{n}^{m}(\cos \theta)}{d \theta} \sin m \phi\right] \\
& H_{\phi}^{T M}= \pm i \omega \alpha_{t_{1}} \sum_{m, n} \frac{b_{o}^{b_{o}} m n}{v_{1}\left(v_{1}+1\right)} z_{v_{1}}^{(q)}\left(k_{t_{1}} r\right) \\
& \times\left[-\epsilon_{\sigma} \frac{m}{\sin \theta} P_{n}^{m}(\cos \theta){ }_{\cos }^{\sin } m \phi\right. \\
& \left. \pm \epsilon_{\tau} \frac{d P_{n}^{m}(\cos \theta)}{d \theta} \sin _{\sin }^{\cos } m \phi\right] \\
& E_{\theta}^{T M}=\alpha_{t_{1}} \sum_{m, n} \frac{b_{o}^{e} m n}{v_{1}\left(v_{1}+1\right)} \frac{\partial\left[r z_{v_{1}}^{(q)}\left(k_{t_{1}} r\right)\right]}{r \partial r} \\
& \times \frac{d P_{n}^{m}(\cos \theta)}{d \theta} \sin _{\sin }^{\cos } m \phi \\
& E_{\phi}^{T M}=\mp \alpha_{t_{1}} \sum_{m, n} \frac{b_{o}^{e} m n}{v_{1}\left(v_{1}+1\right)} \frac{m}{\sin \theta} \frac{\partial\left[r z_{v_{1}}^{(q)}\left(k_{t_{1}} r\right)\right]}{r \partial r} \\
& \times P_{n}^{m}(\cos \theta){ }_{\cos }^{\sin } m \phi \text {. }
\end{aligned}
$$

\section{B. Case 2: $\epsilon_{r} / \mu_{r}=\epsilon_{\tau} / \mu_{\gamma}=\epsilon_{\sigma} / \mu_{\zeta}$}

In this case, the coupled terms in (3) are not zero anymore, which will increase the complexity of obtaining the solutions. Note that $v_{1}=v_{2}$ in (7) if $\epsilon_{r} / \mu_{r}=\epsilon_{\tau} / \mu_{\gamma}$. Hence, to be neat, we let $v=v_{1}=v_{2}$ in the following derivation. Similarly, much effort is made to get the expressions of $E_{r}$ and $H_{r}$, which are bases of obtaining the tangential comppnents of the fields. 
In this case, (5) should be revised into

$$
\left[\begin{array}{l}
E_{r} \\
H_{r}
\end{array}\right]=\sum_{m, n}\left[\begin{array}{l}
E_{n} \\
H_{n}
\end{array}\right] P_{n}^{m}(\cos \theta) \underset{\sin }{\cos } m \phi
$$

In the given relationship between the parameters, with (4) obtained, (7) is reduced to

$$
\begin{aligned}
& \frac{1}{r^{2}} \frac{\partial^{2}\left(r^{2} E_{r}\right)}{\partial r^{2}}-v(v+1) r^{2} E_{r}+\omega^{2} \frac{\epsilon_{\tau}}{\mu_{\gamma}}\left(\mu_{\gamma}^{2}+\mu_{\zeta}^{2}\right) E_{r} \\
& -i \omega \frac{\mu_{r}}{\mu_{\gamma} \epsilon_{r}}\left(\epsilon_{\sigma} \mu_{\gamma}+\epsilon_{\tau} \mu_{\zeta}\right) \frac{1}{r^{2}} \frac{\partial\left(r^{2} H_{r}\right)}{\partial r}=0 \\
& \frac{1}{r^{2}} \frac{\partial^{2}\left(r^{2} H_{r}\right)}{\partial r^{2}}-v(v+1) r^{2} H_{r}+\omega^{2} \frac{\mu_{\gamma}}{\epsilon_{\tau}}\left(\epsilon_{\tau}^{2}+\epsilon_{\sigma}^{2}\right) H_{r} \\
& -i \omega \frac{\epsilon_{r}}{\epsilon_{\tau} \mu_{r}}\left(\epsilon_{\sigma} \mu_{\gamma}+\epsilon_{\tau} \mu_{\zeta}\right) \frac{1}{r^{2}} \frac{\partial\left(r^{2} E_{r}\right)}{\partial r}=0 .
\end{aligned}
$$

We find it feasible to decouple (21) by taking (21a) \pm (21b). After a very lengthy manipulation, we will arrive at two differential equations based on (21a) $\pm(21 b)$ in the form of the Coulomb wave functions, and the solutions of those kinds of differential equations have been solved for in [12] and expressed in terms of cylindrical Bessel functions.

Substituting (20) into (21), we find actually that (21) is associated with $E_{n}$ and $H_{n}$. Finally, with the solutions of the Coulomb wave differential equations in [12], we obtain

$$
E_{n} \pm i \eta_{r}^{2} \eta_{t}^{-1} H_{n}=\beta z_{v}^{(q)}\left(k_{t} r\right) e^{ \pm k_{s} r}
$$

where the interparameters $k_{s}, k_{t}$ and $\beta$ are defined as follows:

$$
\begin{aligned}
k_{s} & =\omega \sqrt{\mu_{\zeta} \epsilon_{\sigma}} \\
k_{t} & =\omega \sqrt{\mu_{\gamma} \epsilon_{\tau}} \\
\beta & =k_{r}^{-\frac{3}{2}} \sqrt{\frac{2 k_{t}}{\pi}} .
\end{aligned}
$$

Hence, we obtain

$$
\begin{aligned}
& E_{n}=\beta \frac{1}{r} z_{v}^{(q)}\left(k_{t} r\right) C h\left(k_{s} r\right) \\
& H_{n}=-i \eta_{r}^{2} \eta_{t}^{-1} \beta \frac{1}{r} z_{v}^{(q)}\left(k_{t} r\right) S h\left(k_{s} r\right) .
\end{aligned}
$$

After obtaining the $E_{n}$ and $H_{n}$, the tangential components of electromagnetic fields can be expressed by following the procedures similar to those in Section A.

\section{Case 3: $\epsilon_{\sigma}=\mu_{\zeta}=0$}

If $\overline{\boldsymbol{\epsilon}}$ and $\overline{\boldsymbol{\mu}}$ bear the uniaxial anisotropic form, namely $\epsilon_{\sigma}=$ $\mu_{\zeta}=0$, field representations were given in [8]. However, the representations of $E_{\phi}^{T M}, H_{\phi}^{T E}$ and $H_{\phi}^{T M}$ are incorrect due to typos. Herein, we present the corrected forms for those items with the notations used by Liu et al.

$$
\begin{aligned}
E_{\phi}^{T M}= & \mp \alpha \sum_{m, n} \frac{{ }_{o}^{e_{o}} m n}{c} v_{1}\left(v_{1}+1\right) \frac{m}{\sin \theta} \frac{\partial\left[r z_{v_{1}}^{(q)}\left(k_{t} r\right)\right]}{r \partial r} \\
& \times P_{n}^{m}(\cos \theta){ }_{\cos }^{\sin } m \phi \\
H_{\phi}^{T E}= & \pm i \eta_{r}^{-2} \eta_{t} \alpha \sum_{m, n} \frac{a_{o}^{e} m n}{v_{2}\left(v_{2}+1\right)} \frac{m}{\sin \theta} \frac{\partial\left[r z_{v_{2}}^{(q)}\left(k_{t} r\right)\right]}{r \partial r} \\
& \times P_{n}^{m}(\cos \theta){ }_{\cos }^{\sin } m \phi \\
H_{\phi}^{T M}= & i \omega \epsilon_{t} \alpha \sum_{m, n} \frac{b_{o}^{e} m n}{v_{1}\left(v_{1}+1\right)} z_{v_{1}}^{(q)}\left(k_{t} r\right) \\
& \times \frac{d P_{n}^{m}(\cos \theta)}{d \theta}{ }_{\sin }^{\cos } m \phi .
\end{aligned}
$$

\section{CONCLUSION}

In this letter, electromagnetic fields in general gyrotropic media are formulated and discussed in spherical systems. Starting from the radial field components, all tangential components of electromagnetic fields are obtained for some cases by means of separation of variables and solving some special differential equations, which lead to the Coulomb wave functions for example. By grouping TE- and TM- wave components together, the fields are finally expressed in a compact form. It is shown that the expressions are reducible to those of a simplier case, i.e., uniaxial anisotropic cases and isotropic cases. The present procedure may be extended to some other cases of more complicated constitutive relations of $\overline{\boldsymbol{\epsilon}}$ and $\overline{\boldsymbol{\mu}}$, which may have more than six parameters.

\section{REFERENCES}

[1] J. A. Kong, Electromagnetic Wave Theory, 2nd ed. New York: Wiley, 1990.

[2] W. C. Chew, Waves and Fields in Inhomogeneous Media. New York: Van Nostrand, 1990.

[3] N. K. Uzunoglu, P. G. Gottis, and J. G. Fikioris, "Excitation of electromagnetic waves in a gyroelectric cylinder," IEEE Trans. Antennas Propag., vol. 33, no. 1, pp. 90-99, Jan. 1995.

[4] J. C. Mozon, "Three-dimensional scattering by an infinite homogeneous anisotropic circular cylinder: a spectral approach," IEEE Trans. Antennas Propag., vol. 35, no. 6, pp. 670-682, Jun. 1987.

[5] J. C. Mozon, "Three-dimensional field expansion in the most general rotationally symmetric anisotropic material: application to scattering by a sphere," IEEE Trans. Antennas Propag., vol. 37, no. 6, pp. 728-735, Jun. 1989.

[6] W. Weiglhofer and W. Paponsek, "Skalare hertz'sche potentiale für gyrotrope medien," AEU: Int. J. Electron. Commun., vol. 39, pp. 343-346, 1985.

[7] W. Weiglhofer, "Field representation in gyrotropic media by one scalar superpotential," IEEE Trans. Antennas Propag., vol. 35, no. 11, pp. 1301-1302, Nov. 1987.

[8] S. Liu, L.-W. Li, M.-S. Leong, and T.-S. Yeo, "Field representations in general rotationally uniaxial anisotropic media using spherical vector wave functions," Microw. Opt. Technol. Lett., vol. 25, no. 3, pp. 159-162, May 2000.

[9] — , "Scattering by an arbitrarily shaped rotationally uniaxial anisotropic object: electromagnetic fields and dyadic Green's functions," Prog. Electromagn. Res., vol. 29, pp. 87-106, 2000.

[10] - "Theory of gyroelectric waveguides," Prog. Electromagn. Res., vol. 29, pp. 231-259, 2000.

[11] L.-W. Li, N.-H. Lim, W.-Y. Yin, and J. A. Kong, "Eigenfunctional expansion of dyadic Green's functions in gy rotropic media using cylindrical vector wave functions," Prog. Electromagn. Res., vol. 43, pp. 101-121, 2003.

[12] W. Magnus and F. Oberhettinger, Formulas and Theorems for the Special Functions of Mathematical Physics. New York: Chelsea, 1949. 